\title{
A UHF RFID Reader Antenna with Tunable Axial Ratio and Fixed Beamwidth
}

\author{
Rui Chen ${ }^{1}$, Shuai Yang ${ }^{1}$, Ajeck M. Ndifon ${ }^{1}$, Ian H. White ${ }^{2}$, Richard V. Penty ${ }^{1}$ and Michael Crisp ${ }^{1}$ \\ ${ }^{1}$ Centre for Photonics Systems, Department of Engineering \\ University of Cambridge, $9 \mathrm{~J}$ J Thomson Ave, Cambridge, CB3 OFA, UK \\ ${ }^{2}$ University of Bath, Claverton Down, Bath BA27AY, UK \\ Email: rc714@cam.ac.uk
}

\begin{abstract}
A novel ultra-high-frequency (UHF) RFID reader antenna is proposed. The antenna has a unique property as being able to change its axial ratio (AR) without affecting its gain, beamwidth or impedance matching performance, enabling the isolated study of the effect of different axial ratios in RFID tag reading.
\end{abstract}

Index Terms-Antennas, Antenna arrays, Radio-frequency identification, UHF antennas, UHF propagation

\section{INTRODUCTION}

Passive ultra-high-frequency (UHF) RFID has become increasingly popular in inventory management and indoor localization [1], [2]. In practical UHF RFID systems, tags are almost always linearly polarized (LP) and randomly oriented [3]. Thus, it is often assumed that, to achieve the best tag read rate, the reader antenna has to be circularly polarized $(\mathrm{CP})$. However, practical experimentation of the effect of imperfect axial ratio is challenging owing to the difficulty of changing axial ratio alone without significantly affecting other antenna parameters. Take the two commercial antennas in Fig. 1 as an example, which are measured in an MVG StarLab [4].

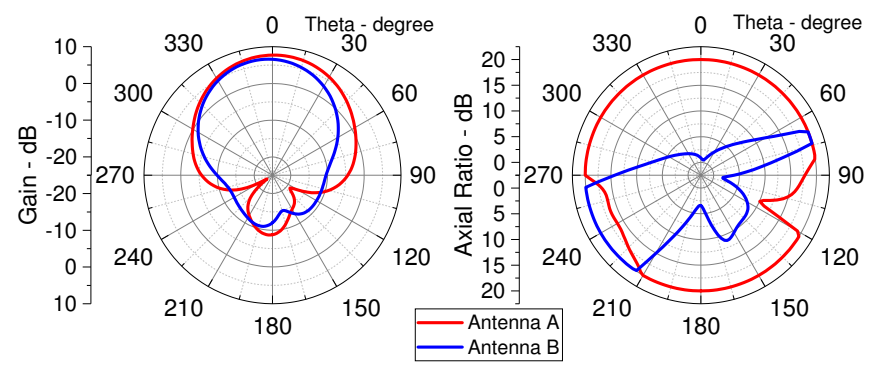

Fig. 1. A comparison of two commercial antennas on beamwidth (left) and axial ratio (right) at $\varphi=0^{\circ}$ cut. Please note that the maximum AR which can be measured by StarLab is $20 \mathrm{~dB}$ and any value above would be cappec [4]

It is clear that their axial ratios are different, however, their gain and beamwidth are also not the same. It would be difficult to evaluate the contribution of axial ratio by directly comparing the two antennas' tag-reading performances as it is impossible to tell how much the performance difference comes from the differences in axial ratio rather than the differences in beamwidth or gain. Thus, an antenna which can change its AR without affecting its beamwidth would be ideal in isolating and studying the effect of AR on a reader antenna's performances.

In [5], we proposed an antenna with two dimensional (2D) beam-steering abilities, a wide AR beamwidth of $136^{\circ}$ and have shown that its AR minima follows its gain maxima. In this work, we show that the antenna is also able to change its axial ratio without affecting the beamwidth or gain.

\section{ANTENNA Design}

\section{A. Antenna Structure}

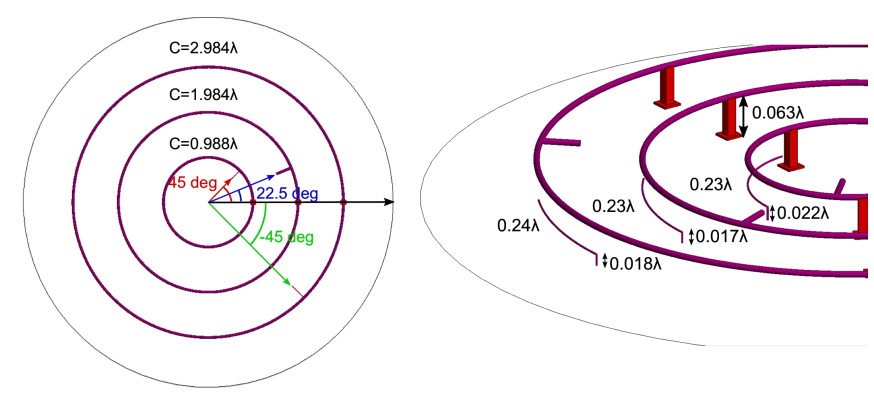

Fig. 2. Structure of the proposed antenna

The structure of the proposed antenna is shown in Fig. 2. The radiating layer of the antenna is composed of three concentric copper loops, each with a perturbation line (PL) to stimulate circular polarization [5], [6], [7]. Changing the angle and length of these perturbation elements would affect the AR performance of the antenna.

A feeding layer is placed between the radiating layer and an aluminium ground plane with a $45 \mathrm{~cm}$ diameter. This layer is formed by three elliptic copper arcs placed directly under three copper loops [5]. Their curvatures are the same as their corresponding copper loops above whereas their lengths and heights from the ground plane can be adjusted to achieve a matched impedance of $50 \Omega$ [5]. The detailed parameters of this antenna are shown in table I.

\section{B. Generating Circular Polarization}

According to [8], a conventional loop antenna with a single feed and a ground plane would be linearly polarized. However, it was discovered in [6] and later analysed in [7] that circular polarization can be stimulated by adding a PL to the loop 
TABLE I

ANTENNA PARAMETERS, WHERE $\lambda$ IS THE WAVELENGTH AT $866 \mathrm{MHz}$ [5]

\begin{tabular}{llll}
\hline & First Ring & Second Ring & Third Ring \\
\hline Perimeter & $0.988 \lambda$ & $1.984 \lambda$ & $2.984 \lambda$ \\
PL Position & $45^{\circ}$ & $22.5^{\circ}$ & $-45^{\circ}$ \\
PL Length & $0.058 \lambda$ & $0.065 \lambda$ & $0.061 \lambda$ \\
Feed line Length & $0.23 \lambda$ & $0.23 \lambda$ & $0.24 \lambda$ \\
Feed line Height & $0.022 \lambda$ & $0.017 \lambda$ & $0.018 \lambda$ \\
\hline
\end{tabular}

with the correct length and position. The current reflected from the PL sums with the current on the loop, changing it from standing-wave-type into travelling-wave-type with a uniform amplitude and a linearly-changing phase as shown in Fig. 3.
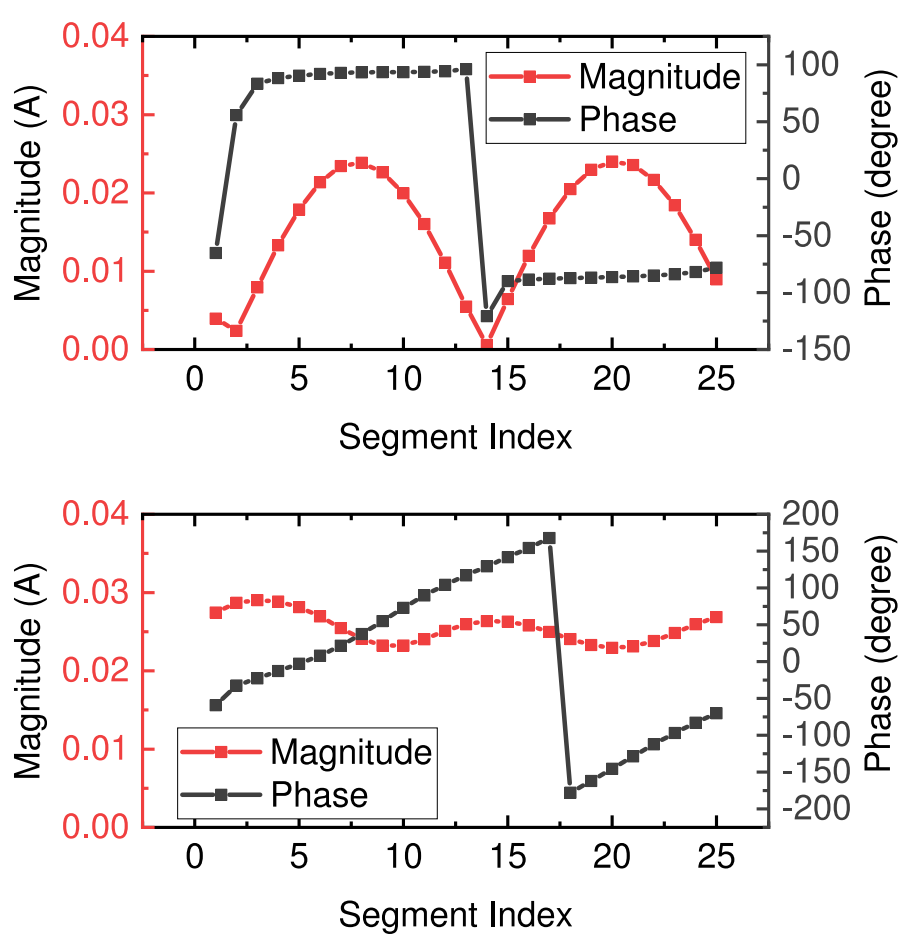

Fig. 3. A comparison of the amplitude and phase distribution of the current on a conventional $1 \lambda$ loop (top) and a $1 \lambda$ loop with a PL (bottom) [5]

As a result, instead of having two current peaks at fixed locations as in conventional loop antennas, in a loop antenna with added perturbation elements, the current peak travels along the antenna. This is analogous to physically rotating the loop antenna around its axis, producing circular polarization [5], [6], [7].

\section{Adjusting AR without Changing Beamwidth}

As has been described in the previous section, the antenna's AR can be adjusted either by adjusting the angle or the length of the perturbation elements. According to [8], the beamwidth and directivity of a loop antenna with a ground plane are mainly affected by the perimeter of the loop and its distance to the ground plane. Thus, it can be assumed that changing the length or the angle of the antenna's PL would only have a minimal effect on the antenna's beamwidth and directivity.

Specifically, since the PL position of the antenna would affect its polarization handedness (by changing the PL position of the antenna from $45^{\circ}, 22.5^{\circ}$ and $-45^{\circ}$ in table I to $-45^{\circ}$, $-22.5^{\circ}$ and $45^{\circ}$, the antenna would change from right hand circular polarization (RHCP) to left hand circular polarization (LHCP) [5], [6], [7]), changing the PL lengths would thus be a better choice than changing PL positions. A series of simulations are conducted using a commercial full-wave simulation software $\left(\mathrm{FEKO}^{\circledR}[9]\right)$.

\section{Simulations}

Take the first loop as an example, in the simulation, the angle of the PL is kept at $45^{\circ}$ as indicated in table I, whereas its length is changed from $1 \mathrm{~mm}$ to $30 \mathrm{~mm}$ in a step of 1 $\mathrm{mm}$. The resulting axial ratio of the antenna is shown in Fig. 4. Note that to speed-up the simulation process, the second and third rings are not included in the simulation, thus the PL length where the AR minima is achieved $(21 \mathrm{~mm})$ and the minimum AR $(1.5 \mathrm{~dB})$ is slightly higher than values shown in [5].

The return loss of the loop is also shown in Fig. 4. From this figure, it is clear that the return loss would be below $-10 \mathrm{~dB}$ when the PL length is between $10 \mathrm{~mm}$ and $23 \mathrm{~mm}$. Correspondingly, this gives an adjustable AR range from 1.5 $\mathrm{dB}$ to $14.3 \mathrm{~dB}$, sufficient for studying the effect of $\mathrm{AR}$ on tag reading performance.

Fig. 5 shows the first loop's beamwidth and gain when the PL length is changed from $10 \mathrm{~mm}$ to $23 \mathrm{~mm}$. Whereas Fig. 6 shows the radiation pattern of the first loop with its PL length at these two extremes. It is clear that neither the antenna's beamwidth nor gain has significantly changed. This verifies our assumption that changing the antenna's PL length would alter the antenna's axial ratio performance but would only have a minimal effect on the antenna's beamwidth and gain.

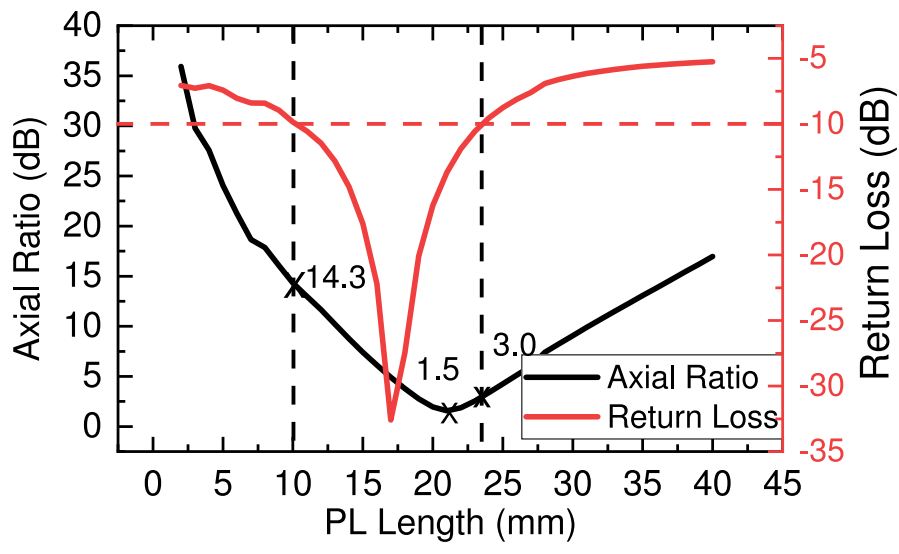

Fig. 4. The simulated axial ratio (the observation point is set at the gain maxima of the loop at $(\theta, \varphi)=(0,0))$ and return loss of the first loop against various PL lengths, with a fixed PL position at $45^{\circ}$ 


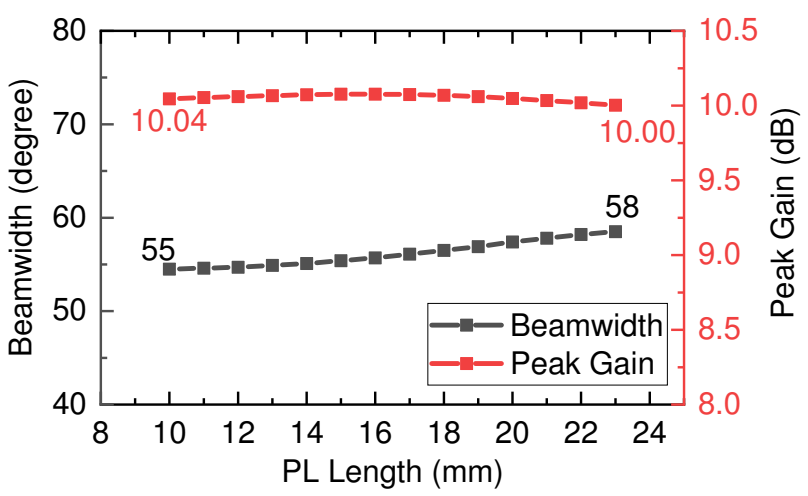

Fig. 5. The simulated beamwidth and gain of the first loop against PL length

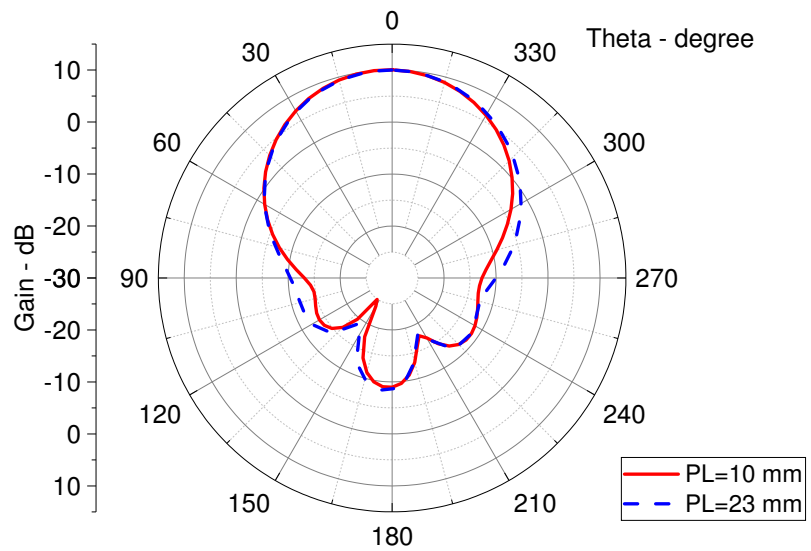

Fig. 6. The simulated radiation pattern of the first loop at $\mathrm{PL}=10 \mathrm{~mm}$ and $\mathrm{PL}=23 \mathrm{~mm}\left(\varphi=0^{\circ}\right.$ cut $)$

\section{Measurements}

To verify the simulation results, the proposed antenna is manufactured and measured using MVG StarLab antenna chamber. A custom phase-shifter board is used for beamsteering. Details of the measurement set-up can be found in [5].

To degrade the antenna's AR performance, the PL length of all three rings are cut by $2.5 \mathrm{~mm}$ compared with the length which minimises AR, and the measured results are shown in Fig. 7 to Fig. 9

It is clear from these results that the antenna's AR ratio is changed from close to $0 \mathrm{~dB}$ to around $4 \mathrm{~dB}$, comparable to a commercial 2-by-2 patch array antenna in [5], whereas its beamwidth and gain are unaffected and its return-loss is still below $-10 \mathrm{~dB}$ in the desired frequency range, covering the lower band UHF RFID frequencies in the European Telecommunications Standards Institute (ETSI) region. Thus, the antenna is suitable to be used in studying the contribution of different AR levels to the RFID tag reading performance.

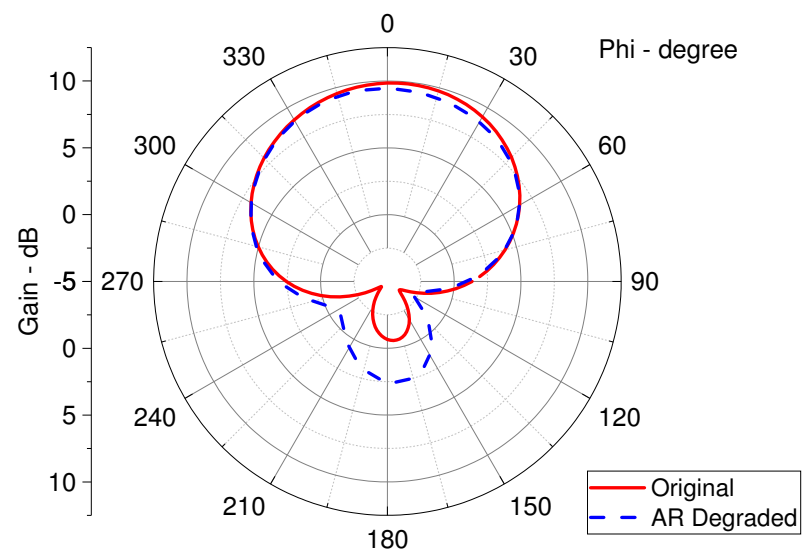

Fig. 7. A comparison of the antenna's measured radiation pattern $\left(\theta=30^{\circ}\right.$ cut)

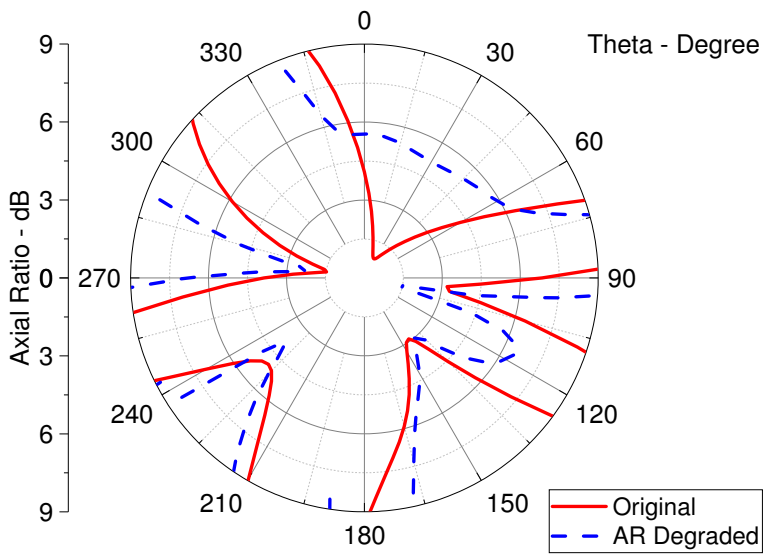

Fig. 8. A comparison of the antenna's measured $\operatorname{AR}\left(\varphi=90^{\circ}\right.$ cut $)$. The antenna's beam is tilted at $\theta=30^{\circ}$

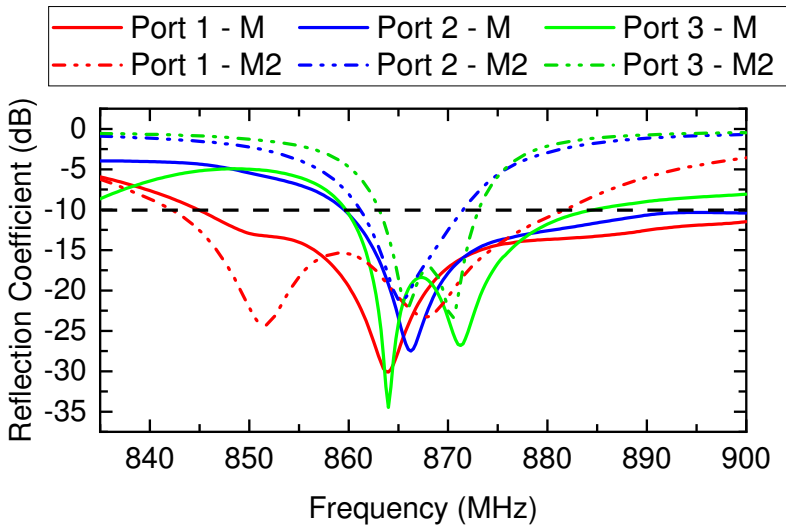

Fig. 9. A comparison of the antenna's measured return loss. M - original design with an optimal AR, M2 - modified design with a degraded AR 


\section{CONCLUSION}

A novel UHF RFID reader antenna is proposed in this work. The antenna is able to change its axial ratio without changing its gain, beamwidth or impedance matching performance. The proposed antenna is suitable for studying the effect of AR independent of other parameters.

\section{ACKNOWLEDGMENT}

This work was supported by EPSRC EP/S-19405/1 Channel Optimised Distributed Passive Sensor Networks.

\section{REFERENCES}

[1] A. P. Sample, D. J. Yeager, P. S. Powledge, A. V. Mamishev, and J. R. Smith, "Design of an RFID-based battery-free programmable sensing platform," IEEE transactions on instrumentation and measurement, vol. 57, no. 11, pp. 2608-2615, 2008.

[2] S. Siachalou, A. Bletsas, J. Sahalos, and A. G. Dimitriou, "RSSI-based maximum likelihood localization of passive RFID tags using a mobile cart," in 2016 IEEE Wireless Power Transfer Conference (WPTC). IEEE, 2016, pp. 1-4.

[3] D. M. Dobkin, The RF in RFID: UHF RFID in practice. Newnes, 2012.
[4] MVG. (2019) StarLab. Accessed on: May 5, 2019. [Online]. Available: https://www.mvg-world.com/en/products/field_product_family/antennameasurement-2/starlab

[5] R. Chen, S. Yang, A. M. Ndifson, I. H. White, R. V. Penty, and M. Crisp, "Beam scanning UHF RFID reader antenna with high gain and wide axial ratio beamwidth," in 2019 IEEE International Conference on RFID Technology and Applications. IEEE, 2019, (To be published).

[6] T. Nakamura and S. Yokokawa, "Loop antenna with a branch wire for circular polarization," Electronics and Communications in Japan (Part I: Communications), vol. 70, no. 11, pp. 110-117, 1987.

[7] H. Nakano, "A numerical approach to line antennas printed on dielectric materials," Computer physics communications, vol. 68, no. 1-3, pp. 441450, 1991.

[8] C. A. Balanis, Antenna theory: analysis and design. John wiley \& sons, 2016.

[9] E. Software and S. Ltd. (2014, May) FEKO user manual. Accessed on: May 5, 2019. [Online]. Available: http://altairuniversity.com/wpcontent/uploads/2015/03/UserManual.pdf

[10] Mini-Circuits. (2019, may) Surface mount phase shifter sphsa152+. Accessed on: May 5, 2019. [Online]. Available: https://ww2.minicircuits.com/pdfs/SPHSA-152+.pdf

[11] qorvo. (2019, may) $5-6000 \mathrm{mhz}$ serial controlled digital step attenuator, 7-bit, $0.25 \mathrm{db}$ steps. Accessed on: May 5, 2019. [Online] Available: https://www.qorvo.com/products/p/RFSA3713 